Article

\title{
Polycondensation of Hexamethylenetetramine in Anhydrous Acid Media as a New Approach to Carbyne-Like Materials and Its Application as Dispersant of Carbon Materials
}

\author{
Alyona V. Gerasimova ${ }^{1}$, Olga V. Alekhina ${ }^{1}$, Leticia García-Cruz ${ }^{2}$, Jesús Iniesta ${ }^{2, *}$, \\ Alexander V. Melezhik ${ }^{1}$ and Aleksey G. Tkachev ${ }^{1}$ \\ 1 Tambov State Technical University, Russian Federation, Sovetskaya str., 106, 392000 Tambov, Russian \\ 2 Department of Physical Chemistry and Institute of Electrochemistry, University of Alicante, \\ 03080 Alicante, Spain \\ * Correspondence: jesus.iniesta@ua.es; Tel.: +34-965909850
}

Received: 31 July 2019; Accepted: 3 September 2019; Published: 6 September 2019

check for updates

\begin{abstract}
Aminocumulene (AC), a technical name that results from the hexamethylenetetramine polycondensation in anhydrous sulfuric acid, was successfully performed toward the synthesis of oligomeric cumulenic compounds made up of carbyne-like $(=C=C)_{x}$ fragments as a main moiety together with amino endcapping groups. The tentative mechanism for the synthesis of the soluble and insoluble AC likely included the participation of carbene species as an intermediate. Spectral properties obtained from UV-Vis, infrared, and Raman spectroscopies, and surface chemistry analysis through X-ray photoelectron spectroscopy of the soluble AC confirmed the presence of a mixture of linear and branched aminocumulene-based oligomers. AC displayed high performance as a dispersant and stabilizer agent of both multi-walled carbon nanotubes and graphene in aqueous solutions after powerful insonation treatment under controlled temperature compared to the most commonly used dispersant agents. Thereby, AC is vitally important for the preparation of carbonaceous materials based on nanoinks in a wide variety of fields.
\end{abstract}

Keywords: carbyne; carbene; aminocumulene; carbon nanotubes dispersion; nanoink

\section{Introduction}

The nature of polymeric substances with a cumulated double-bond chain with both virtually equal double and alternating triple and single carbon-carbon bonds are well-known in the literature [1]. Such structures are assumed for the carbyne that is a new allotropic modification of carbon [2-4]. A review of the synthesis methods for such compounds was reported in [5]. Until now, the question of the existence of this structure of carbyne, with long carbon-carbon chains, is still debatable. However, compounds with short fragments of such chains are synthesized, and their structures are proved by physico-chemical characterization. For example, these compounds contain terminal groups such as alkyl, aromatic, halogenated, or alkoxy groups, depending on the synthesis method [5]. However, the synthesis of carbyne and related compounds is often very complex and unsuitable for upscaling, thereby hampering their practical application.

Cumulene is a linear allotrope of carbon without end-groups and with all carbons connected by double bonds. Moreover, cumulene derivatives have found applications in the field of molecular wires and transistors [6,7], nanoelectronics [8], and non-linear optical materials [9], among others. To fulfill such applications, linear and rigid molecules made up of cumulated carbons connected with a wide variety of electrochemically active functional groups at the end can be synthesized. In this 
regard, Januszewski and Tykwinski have recently reported a wide variety of synthetic approaches, properties, and reactivity of some [n]cumulenes [10]. On the one hand, for an $n$ value equal to 3 , the most standard procedure refers to, in the first instance, the use of the metal-catalyzed dimerization of carbyne/carbenoides [11] with ending caps made of alkanyl or aryl substitutions or, on the other hand, generally using acetylenic diol derivatives using $\mathrm{Li}$ or $\mathrm{Mg}$ acetylides as the precursor, as reported in the review [12].

The novelty of this work relies on the synthesis of the aminocumulene (AC) derivative i.e., containing $\mathrm{H}_{2} \mathrm{~N}-\mathrm{CH}=\mathrm{C}=\mathrm{C}=\mathrm{CH}-\mathrm{NH}_{2}$ as a principal moiety with endcapping amino groups toward the use of an inexpensive and easy scalable dispersing agent. Hence, this work describes the method for producing the aminocumulene, as a technical name, via the polycondensation of hexamethylenetetramine in anhydrous sulfuric acid medium. This work also aims at exploring the physico-chemical features of AC through UV-Vis, Raman, and infrared spectroscopies for the determination of spectral properties, scanning electron microscopy for the assessment of its structure, and X-ray photoelectronic spectroscopy for the examination of the surface chemistry. AC was finally evaluated as dispersant agent of carbon nanotubes and graphene nanoplatelet materials in slight acidic aqueous solutions; then, results were compared to the use of the most common dispersing agents.

\section{Materials and Methods}

\subsection{Reagents and Materials}

Hexamethylenetetramine (HMTA, analytical reagent grade), sulfuric acid (95\%, chemically pure), oleum solution ( $65 \%$, free of sulfur trioxide, chemically pure), and ammonia aqueous solution $\left(25 \% \mathrm{NH}_{3}\right.$, chemically pure) were purchased from Laverna-Lab, Moscow, Russian Federation. Pristine and oxidized multi-walled carbon nanotubes Taunit-M were supplied by NanoTechCenter Ltd. (Tambov, Russian Federation), and natural graphite GCM-2 (>99.5\% purity) was from Zavalivskiy Graphite, Kiev, Ukraine. Enulsifiers Triton X-10 and OP-7 were obtained from ChengduXiYa, Chemical Technology Co. Ltd., Chengdu, China. The rest of the chemical reagents were obtained from the highest purity available. All solutions were prepared using doubly distilled water with resistivity not less than $18.2 \mathrm{M} \Omega \mathrm{cm}$. Sulfuric acid solution containing $5 \mathrm{wt} \%$ oleum solution was prepared by mixing concentrated sulfuric acid with a calculated amount of $65 \mathrm{wt} \%$ oleum.

\subsection{Synthesis of Soluble Aminocumulene under Exhaust Ventilation}

A volume of $60 \mathrm{~mL}$ of $5 \mathrm{wt} \%$ oleum in concentrated sulfuric acid solution was poured into a 2-L glass beaker, and then, $30 \mathrm{~g}$ of HMTA was added stepwise under vigorous stirring, keeping the temperature of reaction mixture near $50{ }^{\circ} \mathrm{C}$. After the complete dissolution of HMTA, the solution was slowly heated up to $85^{\circ} \mathrm{C}$, thereby starting an exothermic reaction with a rising temperature of up to $250^{\circ} \mathrm{C}$ for a short time. It is worth noting that during the reaction mixture, there was a generation of small amounts of sulfur dioxide gas as a result of the side reaction inferred from the reduction of sulfuric acid by organic substances. Thereafter, the reaction mixture was cooled down up to room temperature (rt), after which $400 \mathrm{~mL}$ of water was added to the resulting viscous, almost black solid resin, under stirring condition until complete dissolution. Then, the dark brownish solution was filtered off through a polypropylene microfiber filter, and $200 \mathrm{~mL}$ (excess) of $25 \mathrm{wt} \%$ ammonia aqueous solution was added by keeping the mixture at rt until a precipitate appeared, followed by filtration and further rinsed thoroughly with $0.5 \mathrm{wt} \%$ ammonia aqueous solution until it was almost a colorless filtrate. The final product, with a technical name of aminocomulene (AC), was collected from the filter and dried using an oven at $60{ }^{\circ} \mathrm{C}$ until constant weight. AC solid represents a brilliant, black, fragile material, obtaining a final weight of ca. $8.2 \mathrm{~g}$. We call this product "aminocumulene" (AC), based on its presumable structure (vide infra). The product is insoluble either in neutral and alkaline $\mathrm{pH}$ aqueous solutions or in most common organic solvents (acetone, ethanol, hexzane, benzene, or dimethylsulfoxide, among the most representative solvents), but AC is easily soluble in $1.0 \mathrm{M}$ of acetic 
acid. When the reaction mixture of sulfuric acid and HMTA was processed at higher temperature, the insoluble product was obtained, although the structure of this product was not determined, and it is likely a product from deep polycondensation.

\subsection{Synthesis of Insoluble Product under Exhaust Ventilation)}

A volume of $45 \mathrm{~mL}$ of $5 \mathrm{wt} \%$ oleum in concentrated sulfuric acid solution was poured into a 2-L beaker and then, $30 \mathrm{~g}$ of HMTA was added stepwise under vigorous stirring, keeping temperature of reaction mixture near $50^{\circ} \mathrm{C}$. After the complete dissolution of HMTA, the solution was quickly heated up (flashed mode) to $180-200{ }^{\circ} \mathrm{C}$ for $2 \mathrm{~h}$. After cooling down the reaction mixture up to $\mathrm{rt}, 200 \mathrm{~mL}$ of water was added into the beaker; then, the final product was filtered off and rinsed thoroughly with water, subsequently with $0.5 \mathrm{wt} \%$ ammonia aqueous solution and finally with water. The final result was dried using an oven at $110^{\circ} \mathrm{C}$ until constant weight. The product represents a very hygroscopic black powder (weights vary between $15-16 \mathrm{~g}$ ). Elemental analysis gives $11.1 \%$ content of oxygen and $15.9 \%$ sulfur, with a negligible presence of nitrogen, where sulfur is predominantly in a sulfide form. The mass ratio of oxygen to sulfur is 11.1:15.9 $=0.70$, which is far from the ratio of 1.50 , and is mainly attributed to the presence of $-\mathrm{SO} 3$ groups. This substance exhibits certain interesting properties, which will be explored in a future work.

\subsection{Characterization of $A C$ and Dispersion Preparations}

Sample micrographs and the semiquantitative determination of the elemental composition were performed through the energy-dispersion analysis using a two-beam scanning electron microscope complex Neon 40, Carl Zeiss, Bensheim, Germany. Raman spectra were recorded using a DXR Raman Microscope (ThermoScientific from Germany) with an excitation laser wavelength of $532 \mathrm{~nm}$. X-ray photoelectronic spectroscopy (XPS) experiments were recorded on a K-Alpha Thermo Scientific spectrometer (ThermoScientific from Germany) using Al-K $\alpha(1486.6 \mathrm{eV})$ radiation, monochromatized by a twin crystal monochromator and yielding a focused X-ray spot with a diameter of $400 \mathrm{~mm}$, at $3 \mathrm{~mA}$ $\times 12 \mathrm{kV}$. Processing of the XPS spectra was performed using the Avantage software, with energy values referenced to the $\mathrm{C} 1$ s peak of adventitious carbon located at $284.6 \mathrm{eV}$, and a Shirley-type background.

For the graphite exfoliation and dispersion of carbon nanotubes (CNT), a laboratory ultrasound unit IL-10 was used. The dispersion temperature was kept below $30-35{ }^{\circ} \mathrm{C}$ during the insonation process by cooling the solution using cold water. The optical density of solutions and dispersions was recorded at a KFK-3 spectrophotometer. Concentration of dispersed CNTs was determined spectrophotometrically by recording the optical density of dispersions at $500 \mathrm{~nm}$ after background corrections of the AC solution, using the light absorption coefficient previously found for this batch of CNTs, i.e., $\mathrm{K}=50 \mathrm{~L} / \mathrm{g} \mathrm{cm}$.

\section{Results and Discussion}

\subsection{Synthesis of Soluble and Insoluble AC}

The experiments showed that when using concentrated sulfuric acid $\left(95 \% \mathrm{H}_{2} \mathrm{SO}_{4}\right.$ and $5 \%$ water) as a reaction medium instead of $5 \mathrm{wt} \%$ oleum $\left(95 \% \mathrm{H}_{2} \mathrm{SO}_{4}\right.$ and $\left.5 \% \mathrm{SO}_{3}\right)$, the AC yield (both soluble and insoluble) decreases remarkably. Thus, the absence of water during the polycondensation of HMTA plays an important role for enhancing the AC yield. As it was mentioned previously, the reaction of HMTA polycondensation in anhydrous sulfuric acid or oleum was accomplished with some evolution of gaseous sulfur dioxide and foaming at the reaction mixture. Due to the foaming formation, the maximal loading of the reactor was limited to not more than $45-60 \mathrm{~mL}$ of oleum and $30 \mathrm{~g}$ HMTA per $2 \mathrm{~L}$ of reactor volume. Thus, yield of the process per volume of the reactor was low. We tried to increase the yield by gradually loading the reactor simultaneously with small volumes of reagents (HMTA and $5 \mathrm{wt} \%$ oleum) to the "seed" of the reaction mixture by keeping the temperature near $150{ }^{\circ} \mathrm{C}$. 
Under such conditions, the reaction proceeded calmly, and there was no vigorous foaming formation; nonetheless, the AC yield decreased sharply compared to a single synthesis in the "flash" mode.

Taking into account the complexity of carbyne-like compounds synthesis according to the reported literature [11], we described here a novel, facile chemical procedure using HMTA as a precursor for AC synthesis. As is known, HMTA is usually obtained by the interaction of formaldehyde with ammonia. This reaction is reversible in aqueous media, and when the acid is added, the equilibrium in the aqueous solution is displaced to the opposite direction due to the protonation of ammonia under acidic conditions. However, the protonation of the nitrogen atoms in HMTA under an anhydrous medium at strongly acidic $\mathrm{pH}$ might lead to an increase of the mobility of methylene hydrogen atoms due to the electron density shifting to positively charged protonated nitrogen atoms, thereby prompting apparently unknown chemical reactions.

Formally, the overall reaction (1) could be assumed to explain the synthesis of AC:

$$
\left(\mathrm{CH}_{2}\right)_{6} \mathrm{~N}_{4}+2 \mathrm{H}_{2} \mathrm{SO}_{4} \rightarrow 6 \mathrm{C}+2\left(\mathrm{NH}_{4}\right)_{2} \mathrm{SO}_{4}
$$

Based on the standard thermodynamic data [13] for reagents and products in the hypothetical chemical reaction (1), the standard enthalpy of formation in (1) corresponds to a $-632.4 \mathrm{~kJ} /$ reaction formula when carbon is produced in the form of graphite, and a $-621.6 \mathrm{~kJ} /$ reaction formula when carbon is produced in diamond form. Obviously, the reserve of Gibbs free energy in this reaction is large enough to enable the formation of less stable forms of carbon, and therefore our process is thermodynamically possible. In this regard, our experiments show that in anhydrous sulfuric acid medium, HMTA is capable of undergoing polycondensation with the formation of carbon-like species. Furthermore, upon the stepwise addition of HMTA into $5 \mathrm{wt} \%$ oleum solution under vigorous stirring and cooling conditions with temperatures not higher than $50-60{ }^{\circ} \mathrm{C}$, a colorless solution is formed probably associated to HMTA hydrogen sulfate, which crystallizes upon cooling up to rt. However, if the reaction temperature is raised up to $85-90{ }^{\circ} \mathrm{C}$, an exothermic reaction is initiated, and the temperature increases spontaneously up to $240-250^{\circ} \mathrm{C}$. Under our experimental conditions described in Section 2.2, a black, brown, thin layer of resin is obtained, which is almost a solid at rt. This product dissolves well in water, although depending on the temperature range, it may contain a certain amount of insoluble fraction. Moreover, if after an exothermic "flash" mode condition the reaction mixture is kept for $1-2 \mathrm{~h}$ at $180-200{ }^{\circ} \mathrm{C}$, the polycondensation proceeds far beyond reaching an insoluble black carbon-like product, as mentioned in Section 2.3.

A tentative mechanism is proposed for the synthesis of AC from the polycondensation of HMTA. It can be assumed that during the protonation of nitrogen atoms in HMTA under anhydrous medium at strongly acidic $\mathrm{pH}$, the $\mathrm{C}-\mathrm{N}$ bond can be broken, whereby the protonated nitrogen behaves as an electronegative group. When the protonation of two nitrogen atoms adjacent to one carbon atom takes place, which is possible in strongly acidic medium, the mobility of methylene hydrogens increases, and therefore, they can also be split off. Accordingly, the entire process can be explained by the following mechanism.

$$
\begin{gathered}
>\mathrm{N}-\mathrm{CH}_{2}-\mathrm{N}<+\mathrm{H}^{+} \rightarrow>\mathrm{N}-\mathrm{CH}_{2}-\mathrm{NH}^{+}<,\left(+\mathrm{H}^{+}\right) \leftrightarrow>{ }^{+} \mathrm{HN}-\mathrm{CH}_{2}-\mathrm{NH}^{+}< \\
>{ }^{+} \mathrm{HN}-\mathrm{CH}_{2}-\mathrm{NH}^{+}<\rightarrow>\mathrm{NH}^{+}-\mathrm{CH}:+\mathrm{NH}_{2}{ }^{+}<\leftrightarrow>\mathrm{N}-\mathrm{CH}:+\mathrm{NH}_{2}{ }^{+}<+\mathrm{H}^{+} \\
2>\mathrm{N}-\mathrm{CH}: \rightarrow>\mathrm{N}-\mathrm{CH}=\mathrm{CH}-\mathrm{N}< \\
>\mathrm{N}-\mathrm{CH}=\mathrm{CH}-\mathrm{N}<+\mathrm{H}^{+} \rightarrow>\mathrm{N}-\mathrm{CH}=\mathrm{CH}-\mathrm{NH}^{+}<,\left(+\mathrm{H}^{+}\right) \leftrightarrow>^{+} \mathrm{HN}-\mathrm{CH}=\mathrm{CH}-\mathrm{NH}^{+}< \\
>^{+} \mathrm{HN}-\mathrm{CH}=\mathrm{CH}-\mathrm{NH}^{+}<\rightarrow>{ }^{+} \mathrm{HN}-\mathrm{CH}=\mathrm{C}:+\mathrm{NH}_{2}{ }^{+}<\leftrightarrow>\mathrm{N}-\mathrm{CH}=\mathrm{C}:+\mathrm{NH}_{2}{ }^{+}<+\mathrm{H}^{+} \\
2>\mathrm{N}-\mathrm{CH}=\mathrm{C}: \rightarrow>\mathrm{N}-\mathrm{CH}=\mathrm{C}=\mathrm{C}=\mathrm{CH}-\mathrm{N}<
\end{gathered}
$$


Where step (2) displays the protonation of HMTA by using sulfuric acid: (herein $>\mathrm{N}-\mathrm{CH}_{2}-\mathrm{N}<$ is a fragment of the cyclic structure of HMTA), and step (3) represents the cleavage of the methylene hydrogen and ammonium group in order to form the carbene species. In step (4), the carbene dimerization and further protonation of the dimer takes place in the presence of sulfuric acid, as described in step (5), followed by step (6), which corresponds to the cleavage of the ammonium group from the protonated dimer to form the carbene, with the carbene dimerization finally occurring in step (7). Further chemical reactions involving step (7) can be repeatedly run via the protonation of nitrogen atoms, removal of ammonium ions, and dimerization of the carbene formed in order to increase the length of the cumulene chain. Moreover, carbene species may likely react with $\mathrm{C}=\mathrm{C}$ bonds with the formation of branched polymeric structures.

When the reaction mixture is diluted with water, the acid-catalyzed hydrolysis of unreacted fragments $>\mathrm{N}-\mathrm{CH}_{2}-\mathrm{N}<$ results in the formation of protonated amino groups, according to reaction (8):

$$
>\mathrm{N}-\mathrm{CH}=\mathrm{C}=\mathrm{C}=\mathrm{CH}-\mathrm{N}<+2 \mathrm{H}^{+}+\mathrm{H}_{2} \mathrm{O} \rightarrow{ }^{+} \mathrm{H}_{3} \mathrm{~N}-\mathrm{CH}=\mathrm{C}=\mathrm{C}=\mathrm{CH}-\mathrm{NH}_{3}{ }^{+}\left(+\mathrm{nCH}_{2} \mathrm{O}\right)
$$

In this regard, the resulting oligomeric products dissolve in water at acidic $\mathrm{pH}$ due to the protonation of amino groups. Nonetheless, the addition of ammonia, according to chemical reaction (9) leads to deprotonation of amino groups, and therefore the resulting AC loses its solubility in water:

$$
{ }^{+} \mathrm{H}_{3} \mathrm{~N}-\mathrm{CH}=\mathrm{C}=\mathrm{C}=\mathrm{CH}-\mathrm{NH}_{3}{ }^{+}+2 \mathrm{NH}_{3} \rightarrow \mathrm{H}_{2} \mathrm{~N}-\mathrm{CH}=\mathrm{C}=\mathrm{C}=\mathrm{CH}-\mathrm{NH}_{2}+2 \mathrm{NH}_{4}{ }^{+}
$$

when diluting the acidic reaction mixture with water, an adverse reaction may also occur, with the participation of the tautomers, according to reaction (10).

$$
\mathrm{H}_{2} \mathrm{~N}-\mathrm{CH}=\mathrm{C}=\mathrm{C}=\ldots \leftrightarrow \mathrm{HN}=\mathrm{CH}-\mathrm{HC}=\mathrm{C}=\rightarrow \mathrm{NH}_{4}{ }^{+}+\mathrm{O}=\mathrm{CH}-\mathrm{HC}=\mathrm{C}=\ldots
$$

As a result of the above reaction in an acid medium, nitrogen can be replaced with oxygen to form an aldehyde group. However, in aqueous ammonia medium, reaction (10) can be reversed, as described in reaction (11):

$$
\mathrm{O}=\mathrm{CH}-\mathrm{HC}=\mathrm{C}=\ldots+\mathrm{NH}_{3} \rightarrow \mathrm{H}_{2} \mathrm{O}+\mathrm{HN}=\mathrm{CH}-\mathrm{HC}=\mathrm{C}=\ldots \leftrightarrow \mathrm{H}_{2} \mathrm{~N}-\mathrm{CH}=\mathrm{C}=\mathrm{C}=\ldots
$$

For that reason, AC dissolves first in water containing sulfuric acid, and then is treated with ammonia to precipitate the $\mathrm{AC}$, whereby the product can contain oxygen groups, and therefore its amount in at $\%$ will depend on the synthesis conditions. Accordingly, the final product from the result of HMTA polycondensation can be referred as "aminocumulene". Obviously, this is a technical name, similar to the product itself, which is made of a mixture of oligomers of linear and/or branched structures. The aforementioned proposed mechanism explains why the yield of the HMTA polycondensation is higher when performing the reaction using the "flash mode" rather than under the gradual addition of reagents to the reaction mixture. In this regard, the former synthetic approach will have a greatest concentration of carbene species, thereby prompting the most favorable conditions for its dimerization. Furthermore, we speculate that the amino groups are adjacent to the double bond, i.e., $\mathrm{H}_{2} \mathrm{~N}-\mathrm{CH}=\mathrm{C}=$, although one should consider the possibility of the tautomerization of such a structure through the transition of the hydrogen atom from a nitrogen to a carbon atom, as described in chemical reaction (10).

\subsection{Physicochemical Properties of the AC}

The AC was characterized through SEM, elemental analysis, spectral analysis, and surface chemistry analysis. Figure 1 shows the SEM image at two magnifications of the cleavage surface of the soluble AC, confirming a smooth, homogeneous surface. Table 1 compiles the elemental semiquantitative analysis obtained from the energy-dispersive analysis, demonstrating the presence of 
a high nitrogen at $\%$ as expected, but also the presence of oxygen as inferred from chemical reaction (10) during the AC synthesis. Sulfur is present in a trace amount (not more than $1 \mathrm{wt} \%$ ). Elemental analysis is also in agreement with the XPS data depicted in Figure 2. Carbon, nitrogen, and oxygen core energy levels of $\mathrm{AC}$ confirmed the presence of the above elements. The $\mathrm{C} 1 \mathrm{~s}$ core level spectrum was deconvoluted to various contributions. The intense peak at $284.6 \mathrm{eV}(39.21 \mathrm{at} \%)$ was assigned to carbon in sp2 configurations, the peak at $285.76 \mathrm{eV}\left(22.6\right.$ at \%) was linked to $\mathrm{C}-\mathrm{O}, \mathrm{C}-\mathrm{H}$, and or $\mathrm{H}_{2} \mathrm{~N}-\mathrm{C}$ bonds (phenolic, alkoxy, ether and/or amines), and the contribution featuring at $287.1 \mathrm{eV}(8.31 \mathrm{at} \%)$ was assigned to a $\mathrm{CH}=\mathrm{O}$ bond (carbonyl and/or aldehyde groups). The $\mathrm{O} 1 \mathrm{~s}$ core level signal was decomposed in contributions at $530.8 \mathrm{eV}(7.0 \mathrm{at} \%)$, and was attributed to oxide or/and hydroxide, 532.26 $\mathrm{eV}(4.55 \mathrm{at} \%)$ was assigned to $\mathrm{C}=\mathrm{O}$ bonds (aldehyde/carbonyl), and $533.74 \mathrm{eV}(0.34 \mathrm{at} \%)$ was ascribed to a $\mathrm{C}-\mathrm{O}$ bond (phenol/epoxy/ether). With regard to the $\mathrm{N} 1$ s core energy level, the deconvolution provided the features at a binding energy of $398.39 \mathrm{eV}(7.91$ at $\%)$ linked to the presence of $=\mathrm{CH}-\mathrm{NH}_{2}$ (amine groups), while $399.97 \mathrm{eV}(8.06 \mathrm{at} \%)$ linked to the presence of $\mathrm{C}-\mathrm{N}-\mathrm{C}$ and was attributed to pyrrolic and/or pyridinic groups, and $401.26 \mathrm{eV}(2.03 \mathrm{eV})$ can be attributed to a $\mathrm{C}-\mathrm{N}$ bond, such as for example in quaternary nitrogen species. In general, the observed spectra do not contradict the expected structure of the HMTA polycondensation products. Nonetheless, it cannot be ruled out that other compounds are present in the reaction products, so that the assignment of spectral data cannot be considered to be utterly unambiguous.

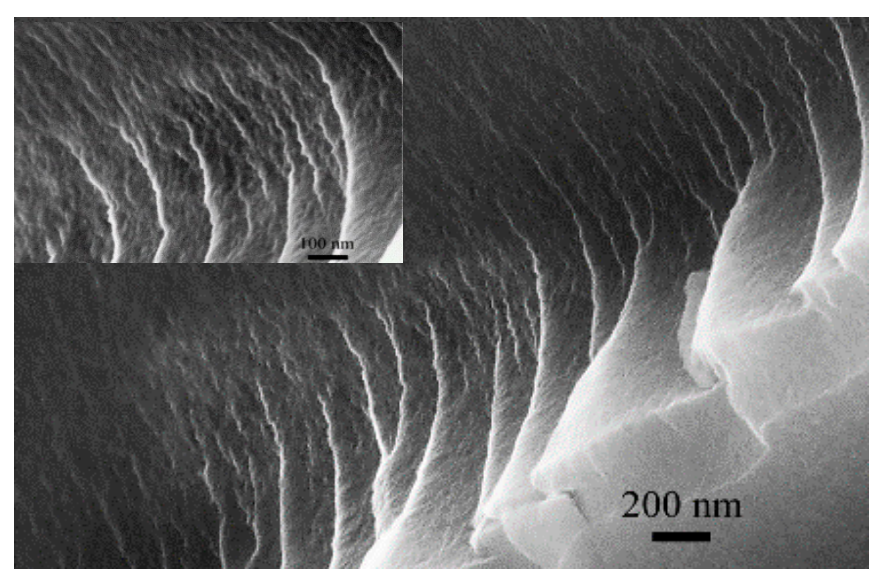

Figure 1. SEM images of the cleavage surface of solid aminocumulene (AC) with different magnifications.

Table 1. Elemental analysis of the solid aminocumulene (AC).

\begin{tabular}{ccc}
\hline Element & $\mathbf{w t} \%$ & at $\%$ \\
\hline $\mathbf{C}$ & $55.43-58.47$ & $60.43-63.48$ \\
& & $(61.96)$ \\
$\mathbf{N}$ & $29.79-27.43$ & $27.85 ; 25.54$ \\
& & $(26.70)$ \\
$\mathbf{O}$ & $13.87-12.85$ & $11.35-10.47$ \\
& & $(10.91)$ \\
$\mathbf{S}$ & $0.91-1.25$ & $0.37 ; 0.51$ \\
& & $(0.44)$ \\
\hline
\end{tabular}

Values between parentheses mean the average value.

With the aim of comparing our results to those in the literature, it is known that the oxygen analogue vinyl alcohol is unstable, because it isomerizes to acetaldehyde. Saebo and Radom [14] performed a quantum mechanical calculation of the vinylamine molecule, which is an analogous moiety of the AC compound synthesized in this work, and predicted the non-planarity of vinylamine with a pyramidal amino group configuration. Nonetheless, vinylamine was prepared by gas-phase 
pyrolysis via aliphatic amines, and the half-life of this molecule was about $10 \min [15,16]$. Additional work performed by Pillsbury and Drucker [17] reported that vinylamine is poorly stable at room temperature, exhibiting a half-life between 2-20 min. Our XPS data are inconsistent with regard to the stability of vinylamine, and hence, we confirm the existence of enamine tautomeric forms. Accordingly, the tautomeric equilibrium (see the chemical reaction number 10) is more likely shifted toward the enamine form, unlike the analogous vinyl alcohol moiety. Thus, the presence of the amine group adjacent to the double carbon-carbon bond upon the structures can be presumably proposed in this work through the polycondensation of HMTA.
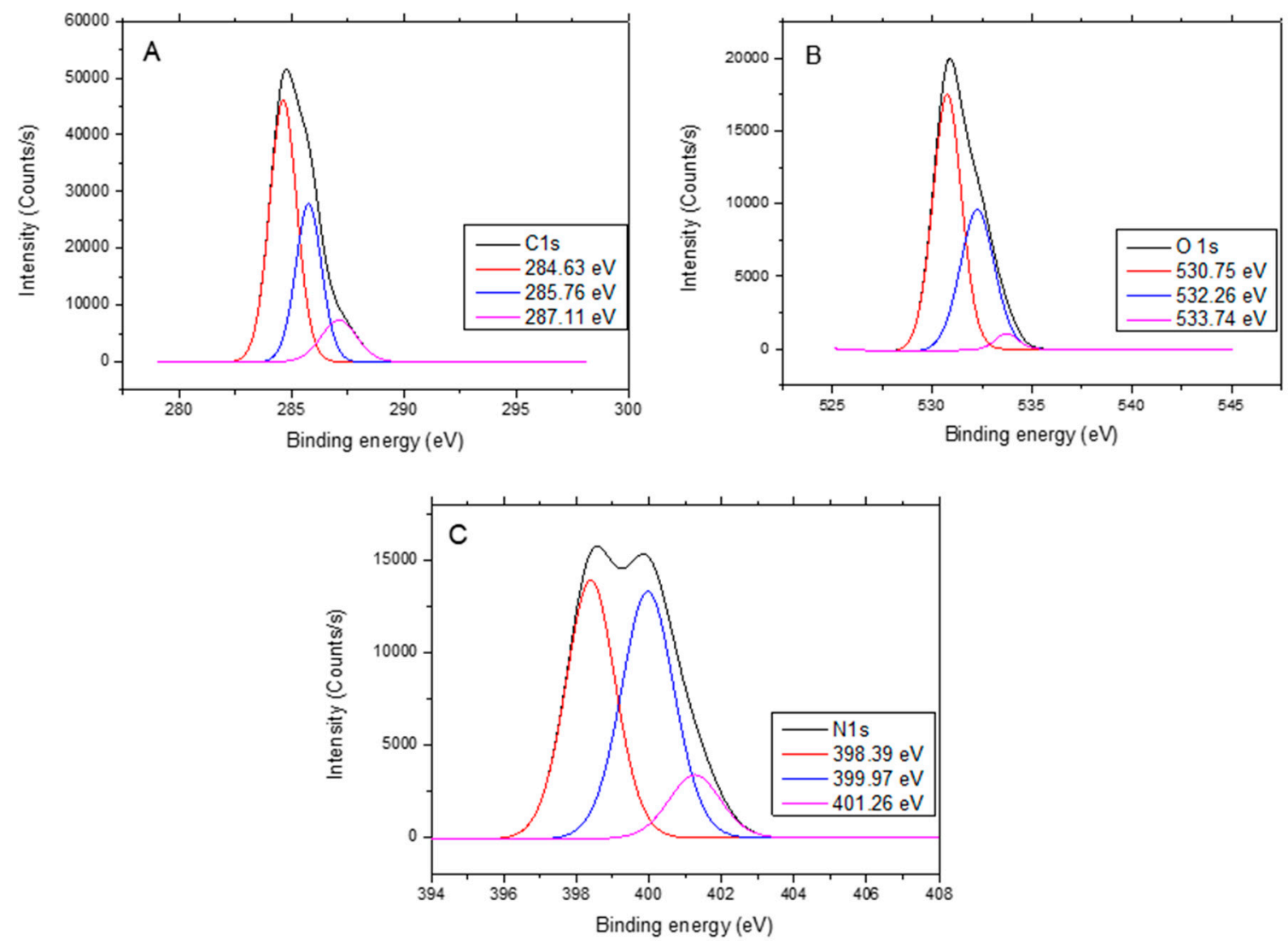

Figure 2. (A) C 1s, (B) O 1s, and (C) N 1s X-ray photoelectronic spectroscopy (XPS) fitted spectra obtained from the surface analysis of solid AC.

Figure $3 \mathrm{~A}$ depicts the UV-Vis absorption spectrum of the AC in $1.0 \mathrm{M}$ of acetic acid, showing a broad band with a maximum peak at $465 \mathrm{~nm}$. In general, the observed spectrum is similar to the absorption spectra of the carbyne-like substances given and synthesized in $[18,19]$. However, the exact correspondence of the UV-Vis spectra cannot be expected, because of all the above molecules are different, connected only by the presence presumably of a carbyne-like fragment of the carbon chain. Nonetheless, it is well established that the factors that govern the UV-Vis spectrum of a cumulene derivative compound include the structure of the [n]cumulene, its length, and the nature of the endcapping groups with mesomeric or inductive properties. In a recent review, Januszewski and Tykwinski [10] stated that even though the maximum electronic absorption is obtained in the UV region (below $400 \mathrm{~nm}$ and dependent on the molecular length), the presence of other electronic absorption peaks is found in the visible region ranging between $420-670 \mathrm{~nm}$, where such values are very dependent on the nature of the endcapping group of the cumulene compound. It is worth stressing that from spectral data reported in [20], the number of pi electrons in our polycumulene chain can be likely estimated in the range between 12-14, i.e., the chain should contain about 11-13 cumulated bonds. 
Furthermore, the presence of maximum absorption of such a high energy, i.e., $465 \mathrm{~nm}$, reflects on the absence of conjugation with the endcapping group and the cumulene [10].
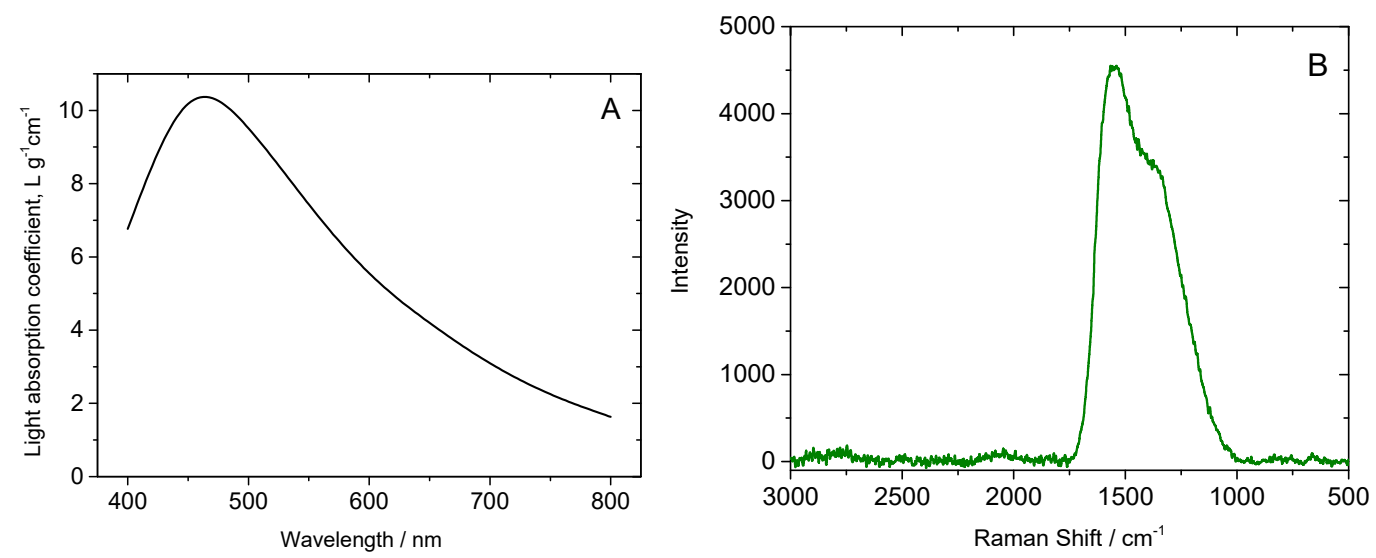

Figure 3. (A) UV-Vis spectrum of AC in $1.0 \mathrm{M}$ of glacial acetic acid; (B) Raman spectrum of solid AC.

In order to justify the UV-Vis optical results with those presented in Table 1, the expected high nitrogen to carbon atomic ratio brings about that the amino group might only be an end-group, but also a bridge group, i.e., two cumulene fragments can be connected through the $-\mathrm{NH}$ - bridge. The role of the amino groups in the $\mathrm{AC}$ as chromophore groups should also be taken into account, as they would lower the energy of the electronic transition. Hence, the observed absorption spectrum agrees with shorter cumulene fragments. Moreover, the presence of branched structures with much lower energy and therefore a decrease in the Highest Occupied Molecular Orbital (HOMO)- Lowest Unoccupied Molecular Orbital (LUMO) energy gap should be likely considered.

Figure 3B shows the Raman spectrum of AC. Two intense overlapping peaks are observed at 1370 and $1570 \mathrm{~cm}^{-1}$, which are characteristic of the low-ordered forms of carbon (usually denoted by D and $\mathrm{G}$ bands, respectively. The broad band featured at $2800 \mathrm{~cm}^{-1}$ can be attributed to vibrations of $\mathrm{N}-\mathrm{H}$ bonds. It is impossible to compare our Raman spectrum to data reported in the literature for various carbyne forms, i.e., polycumulenes and polyynes, because of the very large scattering of literature data, even for the same type of compounds. Hence, the strong influence of substituents on the position and especially the intensity of the bands in the Raman spectrum should be also taken into account.

The 4000-600 $\mathrm{cm}^{-1}$ region of the IR spectrum of the AC compound showed absorption bands at $3156 \mathrm{~s}, 2926 \mathrm{~s}, 2857 \mathrm{~s}, 1631 \mathrm{~s}, 1461 \mathrm{~s}, 1378 \mathrm{~m}, 1092 \mathrm{~s}, 1036 \mathrm{~s}$, and $762 \mathrm{w} \mathrm{cm}^{-1}$. According to the literature [4], cumulated double carbon-carbon bonds are featured by absorption bands in the $1600-1700 \mathrm{~cm}^{-1}$ region. The band at $1631 \mathrm{~cm}^{-1}$ is found within the aforementioned range for polycumulenes. In this case, it is broadened, since the substance is an oligomer, and the lateral $\mathrm{C}=\mathrm{C}$ bonds are in a different environment rather than the $\mathrm{C}=\mathrm{C}$ bonds in the main moiety, although such a band is superimposed on the vibrational band of the carbonyl group, due to the hydrolysis according to chemical reaction (10). A stretching vibrational mode near $2200 \mathrm{~cm}^{-1}$, which is characteristic of the triple carbon-carbon bonds, is absent. A wide stretching vibrational mode at $3456 \mathrm{~cm}^{-1}$ can be attributed to the vibrations of N-H bonds. The bands at $2926 \mathrm{~cm}^{-1}$ and $2857 \mathrm{~cm}^{-1}$ can be attributed to the stretching vibrations of $\mathrm{C}-\mathrm{H}$ bonds, and the band at $1461 \mathrm{~cm}^{-1}$ can be attributed to the bending vibrational mode of $\mathrm{C}-\mathrm{H}$ bonds. For comparative purposes between the AC and HMTA IR spectra, the literature reported the 1300-200 $\mathrm{cm}^{-1}$ region IR spectrum of the HMTA recorded in Nujol showing absorption bands at $1180 \mathrm{w}, 1040 \mathrm{~m}$, $1000 \mathrm{~s}, 823 \mathrm{w}, 803 \mathrm{~s}, 714 \mathrm{w}, 664 \mathrm{~s}, 610 \mathrm{~m}$, and $508 \mathrm{~s} \mathrm{~cm}^{-1}$ [21]. Of these, the bands at 1225 and $1000 \mathrm{~cm}^{-1}$ are assigned to the $\mathrm{C}-\mathrm{N}$ stretching modes. In this regard, our bands at 1092 and $1036 \mathrm{~cm}^{-1}$ can be likely attributed to the $\mathrm{C}-\mathrm{N}$ stretching of the polycomulene compound. 


\subsection{Applications of AC as Dispersant Agent}

The polycumulene AC might exhibit enhanced affinity for the surface of carbon materials, thereby acting as a dispersant and stabilizer of aqueous dispersions containing carbon nanotubes or graphene toward the preparation of nanoinks. In this respect, Figure 4 shows the experimental and theoretical concentration of dispersing pristine and oxidized "Taunit- $\mathrm{M}$ " carbon nanotubes in aqueous acetic acid solution containing AC as dispersing agent. It is worth noticing that an analogous method for the spectrophotometric determination of carbon nanotubes concentration in solutions using various surfactants is described in [22]. According to Figure 4, it can be observed that the best results are achieved with oxidized CNTs compared with the pristine "Taunit-M" CNTs, as shown in Figure 4A,B. Similar results were obtained earlier when the Taunit-M CNTs were dispersed using a water-soluble phenol-formaldehyde resin as a dispersant [23]. As the amount of CNTs increases, the concentration of CNTs in the solution passes through a maximum, which is likely associated with the maximum adsorption of AC dispersant on the CNT surface. By contrast, if the CNT-to-AC ratio becomes too high, the surface concentration of the AC dispersant adsorbed on the CNTs becomes too small to keep CNTs dispersed in solution. However, an increment of the AC starting concentration leads to an increase in the maximum concentration of CNTs in solution, as depicted in Figure 4C. By contrast, the use of traditional surfactants (for example, Triton X-100, sodium dodecylbenzenesulfonate, oil spill dispersant NF, polyvinylpyrrolidone) confirmed that the maximum achievable concentration of CNTs after ultrasonic dispersion in aqueous solution was two orders of magnitude smaller. With respect to the maximum achieved in CNTs concentration, AC as dispersant behaves similar to phenol-formaldehyde resin (PFR) [23]. It is important to highlight several advantages of using AC instead of PFR. Firstly, PFR is used as a dispersant in slightly alkaline medium, while AC is used as a dispersant in acidic $\mathrm{pH}$ medium. Secondly, a significant difference is pointed out when drying CNT dispersions stabilized by PFR, making it impossible to obtain uniform films on surfaces, such as glass plates, because of CNTs' aggregation. In addition, the dispersant aggregation during drying is practically discarded when using $\mathrm{AC}$ as a CNT, making the preparation of thin and uniform films onto different substrates feasible.

Similarly, another use of AC as a dispersant and stabilizer for carbon nanomaterials dispersions was also proven for the exfoliation of graphite. It is known that graphite and various graphitic materials, e.g., thermally expanded graphite or intercalated graphite, split into individual graphene nanoplatelets [24] under the action of ultrasound in aqueous surfactant solutions. However, when using traditional surfactants, only a small fraction of the initial graphite concentration is split into thin nanoplatelets, providing a very low concentration in solution. As a result, the ultrasonic exfoliation of graphite is costly and inefficient. We compared the effectiveness of one of the traditional surfactants OP-7 (the Russian analogue for Triton X-100) and AC in the process of ultrasonic exfoliation of natural graphite GSM-2. The degree of exfoliation was estimated from the optical density of sample dispersions, which, as it was shown in the literature [25], can be an indicator of the thickness of graphene nanoplatelets for comparative analysis. In this regard, Figure 5 depicts the effective light absorption coefficient versus insonation time for graphite exfoliation in the presence of AC and OP-7 as dispersing agents, respectively, providing the former dispersant with the highest performance for dispersions of graphene nanoplatelets. Furthermore, under the same conditions using OP-7, the aggregation of graphene particles after ultrasound switching off occurs so quickly that it is impossible to take a sample for measurement; therefore, data points on the graph are unreproducible. The effectiveness of the use of AC as a dispersant and stabilizer of graphene dispersions nanoplatelets was also confirmed when using the expanded graphite compound (EGC) as a starting graphite material [26].

Figure 6 shows the dependencies of the effective light absorption coefficient at $500 \mathrm{~nm}$ for graphene nanoplatelets produced by the ultrasonic exfoliation of EGC in aqueous solution containing Triton $\mathrm{X}-100$ and $\mathrm{AC}$ in aqueous acetic acid solutions. The results show that the most effective exfoliation of EGC is achieved by using AC with a remarkable graphene concentration of $10 \mathrm{~g} / \mathrm{L}$ ). By contrast, the result of exfoliation using Triton X-100 was worse, even at lower graphene concentrations. 

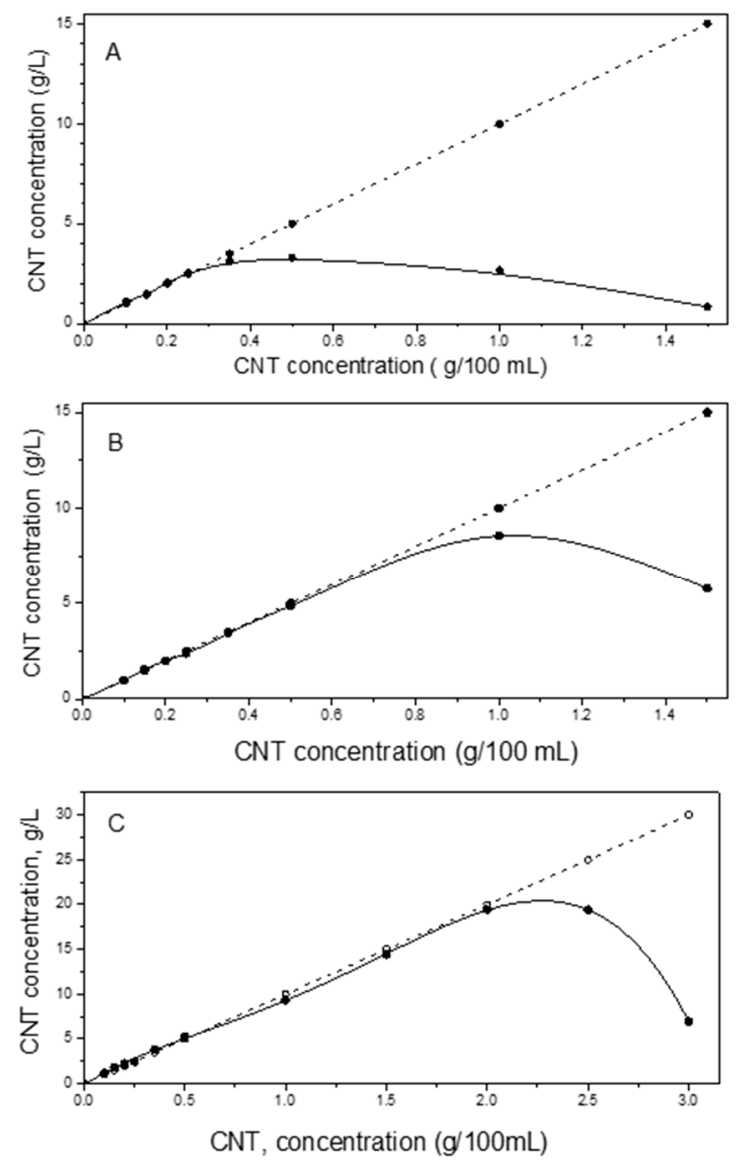

Figure 4. Dispersion of Taunit-M carbon nanotubes (CNTs) in aqueous acetic acid solutions in the presence of AC. Solid lines: real dispersion determined spectrophotometrically; dashed lines: total concentration of CNTs (dispersed and precipitated) in solution. (A) Pristine CNTs with $2.5 \mathrm{~g} / \mathrm{L}$ of AC in $7.5 \mathrm{~g} / \mathrm{L}$ of acetic acid, (B) oxidized CNTs with $2.5 \mathrm{~g} / \mathrm{L} \mathrm{AC}$ in $7.5 \mathrm{~g} / \mathrm{L}$ of acetic acid, (C) oxidized CNTs, with $5.0 \mathrm{~g} / \mathrm{L}$ of $\mathrm{AC}$ concentration in $15.0 \mathrm{~g} / \mathrm{L}$ of acetic acid.

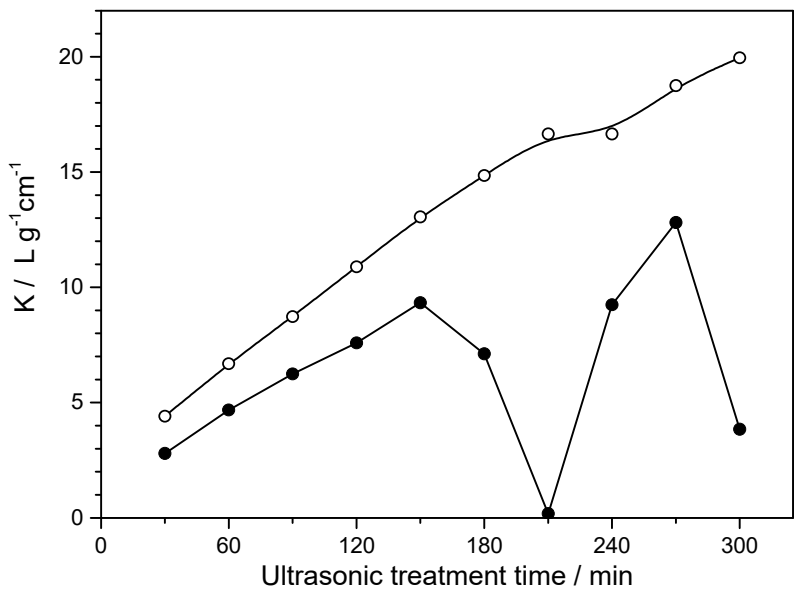

Figure 5. Effective light absorption coefficient $(\mathrm{K}$, at $500 \mathrm{~nm}$ ) versus insonation time for graphite exfoliation in $30.0 \mathrm{~g} / \mathrm{L}$ of aqueous acetic acid solution containing $5.0 \mathrm{~g} / \mathrm{L}$ of AC (open circles), and, $10.0 \mathrm{~g} / \mathrm{L}$ of OP-7 aqueous solution (filling circles). Graphite concentration equals $10.0 \mathrm{~g} / \mathrm{L}$. 


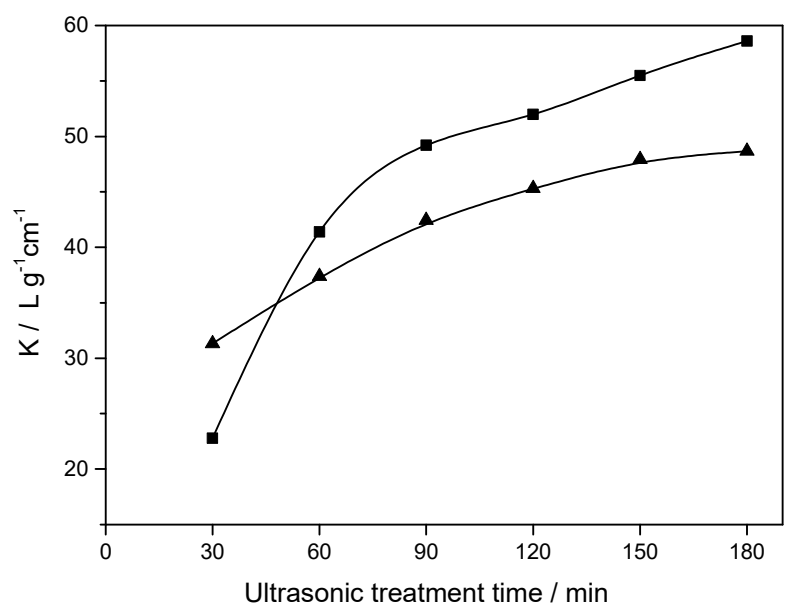

Figure 6. Effective light absorption coefficient (K, at $500 \mathrm{~nm})$ vs. insonation time for expanded graphite exfoliation: in $5.0 \mathrm{~g} / \mathrm{L}$ of AC plus $30.0 \mathrm{~g} / \mathrm{L}$ of acetic acid aqueous solution (squares) and $4.0 \mathrm{~g} / \mathrm{L}$ of Triton $\mathrm{X}-100$ aqueous solution (triangles). Graphite concentration equals $2.0 \mathrm{~g} / \mathrm{L}$.

Thus, AC can find astonishing application in the technology of carbon nanomaterials for the production of nanoinks, thin-film coatings, nanocomposite materials, or in the production of graphene nanoplatelets. Another application of AC as a stabilizer for aqueous dispersions of nanoparticles is the preparation of colloidal solutions of polyaniline. We have found that polyaniline under ultrasonic treatment in aqueous AC containing acetic acid solution provides stable transparent colloidal solutions, thereby obtaining thin transparent films upon drying [Rus Patent RU 2641278].

\section{Conclusions}

The polycondensation of hexamethylenetetramine in anhydrous sulfuric acid was performed for the first time toward the synthesis of the technical product "aminocumulene" (AC). The soluble AC was characterized regarding its topology, spectral features, elemental analysis, and surface chemistry, proving the formation of a mixture of oligomers made up of linear and/or branched structures with amino end-groups. The resulting efficiency of using AC as a dispersing agent of CNTs was dependent on the CNT oxidation grade, and the AC concentration with CNT dispersion concentration values between $0.4-2.0 \mathrm{~g} / 100 \mathrm{~mL}$ in acetic acid solutions. In addition, AC was an effective dispersant and stabilizer of graphene nanoplatelets during the exfoliation of graphite under insonation when compared to other stabilizing agents such OP-7 and Triton X-100. These results are part of the Russian patent RU2661876-C2 [27].

Author Contributions: A.V.M. and A.G.T conceptualized the study and developed the related methodology. A.V.G. and O.V.M. performed the synthesis and characterization of all materials. L.G.-C. and J.I. contributed in terms of XPS and Raman spectroscopy. J.I. and A.V.M provided the discussion of data and the writing of the manuscript. A.G.T. contributed in terms of funding acquisition and project management.

Acknowledgments: The research was carried out at the expense of a grant from the Russian Science Foundation (project No. 15-13-10038). AG acknowledges the Presidential fellowship program of the Ministry of Education and Science of the Russian Federation. Also L.G.-C. and J.I. are grateful to the Spanish MINECO through the project CTQ2016-76231-C2-2-R.

Conflicts of Interest: The authors declare no conflict of interests.

\section{References}

1. Neiss, C.; Trushin, E.; Gorling, A. The Nature of One-Dimensional Carbon: Polyynic versus Cumulenic. Chemphyschem 2014, 15, 2497-2502. [CrossRef]

2. Melnitchenko, V.M.; Nikulin, Y.N.; Sladkov, A.M. Layer chain carbons. Carbon 1985, 23, 3-7. [CrossRef]

3. Banhart, F. Chains of carbon atoms: A vision or a new nanomaterial? Beilstein J. Nanotechnol. 2015, 6, 559-569. [CrossRef] 
4. Stoddart, J.F. The 2rd allotropic form of carbon. Angew. Chem.-Int. Ed. Eng. 1991, 30, 70-71. [CrossRef]

5. Kudryavtsev, Y.P.; Heimann, R.B.; Evsyukov, S.E. Carbynes: Advances in the field of linear carbon chain compounds. J. Mater. Sci. 1996, 31, 5557-5571. [CrossRef]

6. Baughman, R.H. Dangerously seeking linear carbon. Science 2006, 312, 1009-1010. [CrossRef]

7. Akdim, B.; Pachtert, R. Switching Behavior of Carbon Chains Bridging Graphene Nanoribbons: Effects of Uniaxial Strain. Acs Nano 2011, 5, 1769-1774. [CrossRef]

8. Liu, M.; Artyukhov, V.I.; Lee, H.; Xu, F.; Yakobson, B.I. Carbyne from First Principles: Chain of C Atoms, a Nanorod or a Nanorope. Acs Nano 2013, 7, 10075-10082. [CrossRef]

9. Agarwal, N.R.; Lucotti, A.; Tommasini, M.; Chalifoux, W.A.; Tykwinski, R.R. Nonlinear Optical Properties of Polyynes: An Experimental Prediction for Carbyne. J. Phys. Chem. C 2016, 120, 11131-11139. [CrossRef]

10. Januszewski, J.A.; Tykwinski, R.R. Synthesis and properties of long n cumulenes ( $\mathrm{n}>=5)$. Chem. Soc. Rev. 2014, 43, 3184-3203. [CrossRef]

11. Stang, P.J.; Fox, D.P.; Collins, C.J.; Watson, C.R. Unsaturated carbenes from primary vinyl triflates. 9. Intramolecular rearrangement via free carbenes. J. Org. Chem. 1978, 43, 364-365. [CrossRef]

12. Bildstein, B. Cationic and neutral cumulene sp-carbon chains with ferrocenyl termini. Coord. Chem. Rev. 2000, 206, 369-394. [CrossRef]

13. Lide, D.R. CRC Handbook of Chemistry and Physics: A Ready-reference Book of Chemical and Physical Data; CRC-Press: New York, NY, USA, 1995.

14. Saebo, S.; Radom, L. The structure of vinylamine. J. Mol. Struct.-Theochem 1982, 6, 227-233. [CrossRef]

15. Brown, R.D.; Godfrey, P.D.; Kleibomer, B.; Pierlot, A.P.; McNaughton, D. Submillimiter wave spectrum, far infrared spectrum, and inversion potential of vinylamine. J. Mol. Spectrosc. 1990, 142, 195-204. [CrossRef]

16. Hamada, Y.; Hashiguchi, K.; Tsuboi, M.; Koga, Y.; Kondo, S. Pyrolysis of amines -Infrared spectrum of vinylamine. J. Mol. Spectrosc. 1984, 105, 93-104. [CrossRef]

17. Pillsbury, N.R.; Drucker, S. Ultraviolet spectrum of vinylamine. J. Mol. Spectrosc. 2004, 224, 188-193. [CrossRef]

18. Cataldo, F. A study on the structure and electrical properties of the fourth carbon allotrope: Carbyne. Polym. Int. 1997, 44, 191-200. [CrossRef]

19. Kastner, J.; Kuzmany, H.; Kavan, L.; Dousek, F.P.; Kurti, J. Reductive preparation of carbynewith high yied-An in situ raman scatteringstudy. Macromolecules 1995, 28, 344-353. [CrossRef]

20. Berlin, A.A.; Cherkash, M. Effective conjugation and structure polyconjugatedmacromolecules. Vysokomolekulyarnye Soedineniya Sect. A 1971, 13, 2298.

21. Ahuja, I.S.; Singh, R.; Yadava, C.L. Structural information on cobalt(II), nickel(II), copper(II), zinc(II), silver(II) and cadmium(II) nitrate complexes with hexamethylenetetramine from their magnetic moments, electronic and infrared spectra. J. Mol. Struct. 1980, 68, 333-339. [CrossRef]

22. Rastogi, R.; Kaushal, R.; Tripathi, S.K.; Sharma, A.L.; Kaur, I.; Bharadwaj, L.M. Comparative study of carbon nanotube dispersion using surfactants. J. Colloid Interface Sci. 2008, 328, 421-428. [CrossRef]

23. Burakova, E.; Melezhyk, A.V.; Gerasimova, A.; Galuninm, E.; Memeto, N.; Tkachev, A.G. A New Way of Developing Nanocomposites Based on Carbon Nanotubes and Graphene Nanoplatelets. Nanopages 2016, 11, 1-11. [CrossRef]

24. Grayfer, E.D.; Makotchenko, V.G.; Nazarov, A.S.; Kim, S.J.; Fedorov, V.E. Graphene: Chemical approaches to the synthesis and modification. Russ. Chem. Rev. 2011, 80, 751-770. [CrossRef]

25. Melezhyk, A.V.; Kotov, V.A.; Tkachev, A.G. Optical Properties and Aggregation of Graphene Nanoplatelets. J. Nanosci. Nanotechnol. 2016, 16, 1067-1075. [CrossRef]

26. Melezhyk, A.V.; Tkachev, A.G. Synthesis of graphene nanoplatelets from peroxosulfate graphite intercalation compounds. Nanosyst. Phys. Chem. Math. 2014, 5, 294-306.

27. Tkachev, A.G.; Melezhik, A.V.; Alekhina, O.V. New Cumulene Substance Useful for Producing Nitrogen-Containing Carbon Films, and for Dispersing and Exfoliating Graphite Materials, Comprises Chain of Cumulated Double Carbon-Carbon Bonds, and Has End Groups Comprising Amino Groups. Russia Patent RU2661876-C2, 20 July 2018.

(C) 2019 by the authors. Licensee MDPI, Basel, Switzerland. This article is an open access article distributed under the terms and conditions of the Creative Commons Attribution (CC BY) license (http://creativecommons.org/licenses/by/4.0/). 